\title{
UTILIZAÇÃO DO TRANSPORTE ATIVO PARA AUMENTO DA QUALIDADE DE VIDA DA POPULAÇÃO URBANA DURANTE A PANDEMIA DO COVID-19 À LUZ DE ABORDAGEM BIBLIOMÉTRICA
}

\author{
USE OF ACTIVE TRANSPORTATION TO INCREASE THE QUALITY OF LIFE OF THE URBAN \\ POPULATION DURING THE COVID-19 PANDEMIC IN THE LIGHT OF A BIBLIOMETRIC
}

\section{APPROACH}

\section{$\underline{\text { Victor Hugo Souza de Abreu }}{ }^{1 *}$, Isabella Martins de Almeida ${ }^{2} \& \underline{\text { Larissa Rodrigues }}$ $\underline{\text { Turini }}^{3}$}
${ }^{123}$ Instituto Alberto Luiz Coimbra de Pós-Graduação e Pesquisa de Engenharia da Universidade Federal do Rio de Janeiro (COPPE/UFRJ)
1* victor@pet.coppe.ufrj.br ${ }^{2}$ isabellamartins@pet.coppe.ufrj.br ${ }^{3}$ larissa.turini@gmail.com

\section{ARTIGO INFO.}

Recebido em: 23.06.2021

Aprovado em: 12.07.2021

Disponibilizado em: 23.08.2021

Palavras-Chave: Transporte ativo; Pandemia; COVID-19; Saúde física; Saúde mental.

KEYWORDS: Active transportation; Pandemic; COVID-19; Physical health; Mental health.

*Autor Correspondente: Abreu, V. H. S.

\section{RESUMO}

As restrições implementadas pelos governos para conter a disseminação do coronavírus (COVID-19), incluindo distanciamento social, isolamento e confinamento domiciliar, tiveram grande impacto na qualidade de vida da população urbana. Nesse sentido, a utilização do transporte ativo passou a configurar-se como uma importante estratégia para que a população tivesse acesso aos bens essenciais, permitindo uma maior distância entre os indivíduos, bem como para melhorar aspectos relacionados a saúde física e mental durante o período de pandemia. Servindo a esse propósito, este artigo tem como objetivo realizar uma revisão bibliográfica com abordagem bibliométrica para identificar como o transporte ativo pode influenciar na qualidade de vida da população urbana durante o período de pandemia. Os resultados indicam que a investigação sobre o assunto, embora prematura, encontra-se em crescimento, sendo publicados estudos em importantes periódicos internacionais. Além disso, as discussões levantadas aqui mostram que o transporte ativo corresponde a um modo de transporte crítico que necessita de um tratamento distinto, mais atento as demandas da população e das necessidades financiamento, tanto durante períodos normais, quanto em grandes perturbações sociais, como é o caso de uma pandemia como a do COVID-19.

\section{ABSTRACT}

The restrictions implemented by governments to curb the spread of the coronavirus (COVID-19), including social distance, isolation and home confinement, had a major impact on the quality of life of the urban population. Thus, the use of active transportation started to be configured as an important strategy so that the population had access to essential goods, allowing a greater distance between individuals, as well as to improve aspects related to physical and mental health, during the period pandemic. Serving this purpose, this article aims to carry out a bibliographic review with a bibliometric approach to identify how active transportation can positively influence the quality of life of the urban population during the pandemic period. The results indicate that research on the subject, although premature, is growing, and studies have been published in important international journals. In addition, the discussions raised here show that active transportation corresponds to a critical mode that deserves more attention and funding, both during normal periods and in major social disruptions, such as a pandemic such as COVID-19. 
Citação (APA): Abreu, V. H. S., de, Almeida, I. M., de, \& Turini, L. R. (2021). Utilização do transporte ativo para aumento da qualidade de vida da população urbana durante a pandemia do Covid-19 à luz de abordagem bibliométrica. Brazilian Journal of Production Engineering, $7(3), 139-152$

\section{INTRODUÇÃO}

O transporte ativo pode ser amplamente definido como uma variedade de modos de transporte movidos por propulsão humana (Alfonsin et al., 2019), incluindo, caminhadas e ciclismo, principalmente, e outros menos usuais tais como patins em linha, patinetes, skate e viagens com o uso de um auxílio à mobilidade, como uma cadeira de rodas (Chan \& Farber, 2019).

A promoção do transporte ativo, especialmente em áreas de densa urbanização, ao longo dos últimos anos tornou-se tema central das discussões relacionadas às políticas públicas (Sallis $e t$ al., 2004; de Nazelle et al., 2011; Rodrigues et al., 2019), despontando-se como uma valiosa alternativa de mobilidade urbana sustentável (Beck \& Hensher, 2020; Abreu \& Turini, 2021; Santos et al., 2021; Almeida \& Abreu, 2021). Especificamente sobre o ponto de vista social, o transporte ativo contribui significativamente para melhoria da qualidade de vida de seus praticantes, pois une a saúde ao bem-estar (Rojas-Rueda et al., 2016; Giménez-Gaydou et al., 2019; Alfonsin et al., 2019), além de proporcionar prazer, flexibilidade e liberdade ao usuário (Rissel et al., 2015).

Nesse sentido, o transporte ativo constitui-se de uma importante estratégia para manter a saúde física e mental de seus praticantes, principalmente durante períodos de isolamento social, estabelecidos com o objetivo de conter a disseminação do COVID-19 (NCMM, 2020). $\mathrm{O}$ isolamento por um longo período ou confinamento em casa pode causar efeitos negativos na saúde psicossocial e mental do indivíduo, causando, especialmente, estresse, emoções negativas e cognição prejudicada (Hawkley \& Capitanio, 2015).

Dessa maneira geral, as atividades relacionadas ao transporte ativo podem minimizar esses problemas, aumentando a qualidade de vida da população urbana e promovendo bem estar social nestes períodos de distanciamento, afinal essas atividades geralmente são realizadas em ambientes amplos e ao ar livre, o que permite uma maior distância entre os indivíduos, tanto durante a prática de atividades físicas quanto no deslocamento entre pontos de origem e destino (quando comparado ao transporte público, por exemplo), para fins de específicos, como deslocamento casa-trabalho, evitando aglomerações (BCHLA, 2020) e reduzindo o risco de desenvolvimento de várias doenças (Wannmacher, 2016; Brooks et al., 2020a) como a ansiedade e o estresse (Brady et al., 2020).

Portanto, este artigo tem como objetivo apresentar um apanhado geral de estudos aplicáveis a utilização do transporte ativo para aumento da qualidade de vida da população urbana, principalmente, quanto ao aumento da saúde física e mental, durante a pandemia do COVID19, por meio de uma revisão da literatura, que utiliza criteriosos filtros de inclusão e qualificação de estudos, com abordagens bibliométricas. Destaca-se que as buscas englobam a base de dados Web of Science, de alcance e cobertura satisfatórios, de modo a obter uma maior quantidade de estudos condizentes com a pesquisa.

Cabe destacar ainda que, embora aspectos não relacionados aos transportes sejam considerados, em sua maioria, serviços essenciais, tais como o desenvolvimento de tratamentos, testes e vacinas, os estudos focados nos transportes apresentam um papel

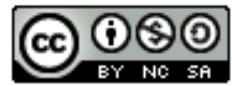


fundamental na mitigação dos efeitos negativos da pandemia atual e eventos perturbadores futuros, incluindo a possível disseminação de novas doenças (Hendrickson \& Rilett, 2020).

Para alcançar seus objetivos, além dessa seção introdutória, este estudo está estruturado da seguinte forma: a Seção 2 apresenta e descreve o procedimento metodológico; a Seção 3 apresenta e discute os resultados bibliométricos; a Seção 4 expõe uma visão geral sobre como o transporte ativo pode favorecer o aumento da saúde física e mental durante a pandemia do COVID-19; e, finalmente, a Seção 5 contém as considerações finais.

\section{PROCEDIMENTO MEDODOLÓGICO}

Este estudo adota como procedimento metodológico uma revisão da literatura com abordagens bibliométricas que busca apresentar uma visão geral de estudos que investigam como o transporte ativo pode melhorar a saúde física e mental durante a pandemia do COVID-19, bem como favorecer a atividade física em tempos de lockdown (Figura 1).

Figura 1. Procedimento metodológico.

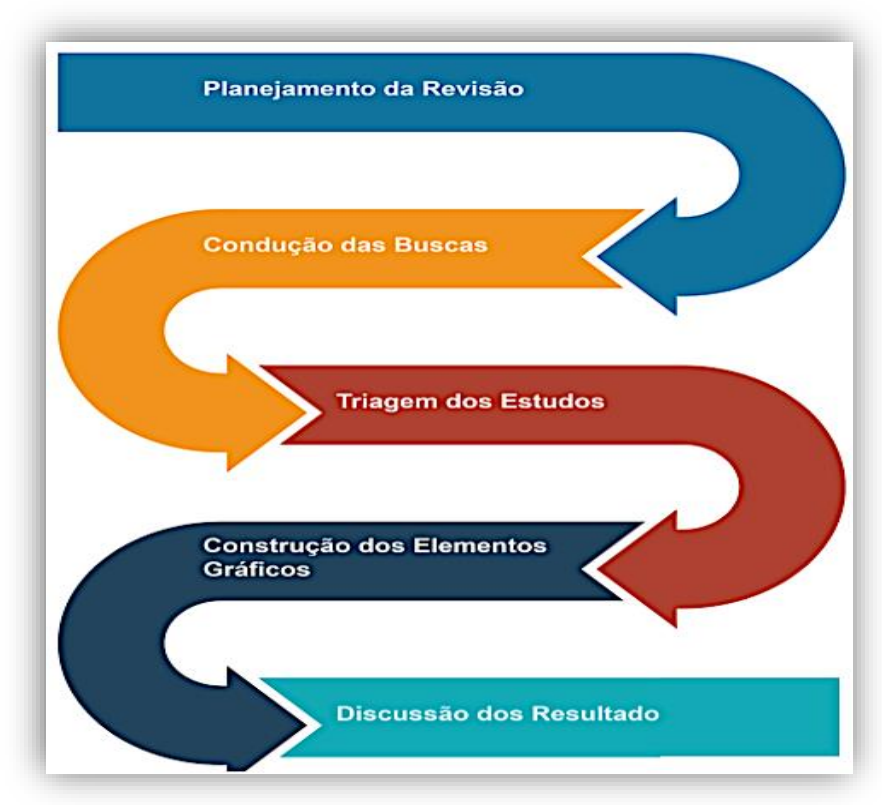

Fonte: Autores (2021).

O planejamento da revisão engloba a definição das estratégias de busca. Nesse sentido, os termos utilizados, critérios de inclusão e qualificação, bem como os detalhes para a busca e extração do banco de dados, são apresentados na Tabela 1, em que TS = Tópico, que significa as palavras que são pesquisadas nos títulos, resumos e palavras-chave dos artigos.

Tabela 1. Descrição das Estratégias de Buscas.

\begin{tabular}{|c|c|}
\hline Critério & Descrição \\
\hline Tópicos & $\begin{array}{c}\text { TS }=(" \text { active transport } * " \text { AND "COVID-19") OR TS }=(" \text { active transport*" AND } \\
\text { "coronavirus") OR TS=("active travel" AND "COVID-19") OR TS=("active travel" } \\
\text { AND "coronavirus") OR TS = ("cycling" AND "COVID-19") OR TS = ("cycling" } \\
\text { AND "coronavirus") OR TS }=(" \text { walking" AND "COVID-19") OR TS = ("walking" } \\
\text { AND "coronavirus") }\end{array}$ \\
\hline Base de Dados & Web of Science \\
\hline Método de Busca & Busca Direta \\
\hline Indexes & SCI-EXPANDED, SSCI, A\&HCI, CPCI-S, CPCI-SSH, ESCI \\
\hline Inclusão & $\begin{array}{l}\text { (I) Tempo de cobertura: todos os anos da base de dados }(1945-2021) \text {; } \\
\text { (c) (1) }\end{array}$ \\
\hline
\end{tabular}


Citação (APA): Abreu, V. H. S., de, Almeida, I. M., de, \& Turini, L. R. (2021). Utilização do transporte ativo para aumento da qualidade de vida da população urbana durante a pandemia do Covid-19 à luz de abordagem bibliométrica. Brazilian Journal of Production Engineering, $7(3), 139-152$

Critério

\section{Descrição}

(II) Enquadramento com o objetivo proposto; (III) Fator de impacto do periódico; e (IV) Tipos de documentos: somente artigos.

(I) A pesquisa apresenta uma revisão bibliográfica bem fundamentada? (II) O estudo apresenta inovação técnica? (III) As contribuições são discutidas? (IV) As limitações são explicitamente declaradas? e (V) Os resultados e conclusões são consistentes com os objetivos pré-estabelecidos? 08 de março de 2021, as $19 \mathrm{~h} 00 \mathrm{~min}$

Fonte: Autores (2021).

Na condução das buscas, realizada no início de março de 2021, os termos de pesquisa foram inseridos na base de dados Web of Science, já considerando alguns critérios de inclusão como presença de apenas artigos e ano de cobertura, que podem ser selecionados pelos filtros da base. Salienta-se que se procurou conseguir uma certa abrangência na escolha dos termos para ampliar o campo de busca e assim obter um maior número de estudos condizentes com a pesquisa. A triagem de artigos incluiu duas etapas: (i) aplicação dos critérios de inclusão faltantes, para uma triagem preliminar; e (ii) aplicação dos critérios de qualificação, para uma triagem final. Dessa forma, de 130 artigos incluídos incialmente, apenas 38 publicações atenderam a todos os critérios de inclusão e de qualificação.

$\mathrm{Na}$ construção dos recursos gráficos, utilizaram-se algumas ferramentas tais como Excel, Plataforma Bing, Vos Viewer, dentre outros. Essas ferramentas permitem auxiliar o processo de visualização dos resultados de forma clara e direta, bem como tornar o estudo mais representativo e dinâmico. Por fim, na discussão dos resultados, são expostas as análises bibliométricas considerando os estudos/artigos incluídos no repositório de pesquisa, bem como também é realizadas uma revisão geral (overview) dos principais pontos que norteiam a utilização do transporte ativo no combate a pandemia do COVID-19.

Menciona-se que, embora o estudo tenha utilizado apenas o Web of Science, não considerando publicações contidas em outras bases de dados como ScienceDirect, Scielo e Scopus, a base escolhida para realização das buscas apresenta alcance e cobertura satisfatórios (Chen, 2010). Além disso, é importante destacar que os termos de busca podem influenciar nos resultados, portanto, os artigos incluídos no repositório de pesquisa são limitados pelo banco de dados e os termos de busca utilizados.

\section{ANÁLISES BIBLIOMÉTRICAS}

A partir da busca realizada na base Web of Science, foi possível verificar que 38 publicações estavam aptas a serem incluídas no repositório de pesquisa, ou seja, atendiam aos critérios de inclusão e qualificação (qualidade e aplicabilidade) definidos previamente.

Desses estudos, em relação a evolução das publicações ao longo dos anos, como a pandemia do COVID-19 teve início em dezembro de 2019, na China, e teve impactos significativos no restante do mundo apenas em 2020, o número de publicações concentra-se no ano de 2020, que corresponde ao ano de publicação de quase $90 \%$ dos artigos. O restante das publicações (10\%) foi publicado no ano corrente de 2021. Cabe mencionar que, o fato de o assunto ser novo e continuar em pauta, desperta o interesse de novos pesquisadores, bem como pode concretizar estudos já em andamento, que até o final do ano de 2021 podem inclusive ultrapassarem a quantidade de estudos de 2020. 
Citação (APA): Abreu, V. H. S., de, Almeida, I. M., de, \& Turini, L. R. (2021). Utilização do transporte ativo para aumento da qualidade de vida da população urbana durante a pandemia do Covid-19 à luz de abordagem bibliométrica. Brazilian Journal of Production Engineering, $7(3), 139-152$.

Torna-se também pertinente avaliar os artigos por periódico de publicação, de modo a identificar quais são as revistas que se interessam pelo assunto, bem como o fator de impacto de cada uma delas. Isso permite que pesquisadores direcionem seus esforços de publicação para periódicos que se interessam diretamente pelo assunto estudado, evitando perder tempo desnecessário de submissão em revistas não tratam do assunto averiguado e, que, consequentemente, rejeitariam a publicação.

Nesse sentido, a Tabela 2 apresenta os periódicos ordenados por número de publicações sobre $\mathrm{o}$ assunto (primeiro critério) e fator de impacto (segundo critério). Destaca-se que $\mathrm{P}$ (Publicações) refere-se a porcentagem de artigos publicados no periódico sobre a área de interesse investigada e FI (Fator de Impacto) avalia a importância de periódicos científicos em suas respectivas áreas com valores correspondentes ao ano de 2019.

Tabela 2. Publicações por Periódico.

\begin{tabular}{ccc}
\hline Periódico & P & FI \\
\hline International Journal of Environmental & $13 \%$ & 2,849 \\
Research and Public Health & $5 \%$ & 2,067 \\
Frontiers in Psychology & $5 \%$ & 4,419 \\
International Journal of Obesity & $5 \%$ & 2,576 \\
Sustainability &
\end{tabular}

Fonte: Autores (2021).

$\mathrm{Na}$ Tabela 2, nota-se que o periódico que mais aborda sobre o assunto é o International Journal of Environmental Research and Public Health, que é um periódico científico interdisciplinar de acesso aberto revisado por pares, publicado pela Multidisciplinary Digital Publishing Institute (MDPI). Além disso, outros três periódicos se destacam, com 5\% das publicações cada, que são: o Frontiers in Psychology, o International Journal of Obesity e o Sustainability. O restante das publicações encontra-se pulverizado em outros 27 periódicos.

Considera-se pertinente avaliar também os países de origem das instituições de ensino dos autores que mais desenvolvem artigos relevantes sobre o assunto, ou seja, trata-se de uma identificação da origem/base de trabalho dos pesquisadores. Isso permite mostrar os países que mais se interessam sobre a temática, bem como demonstrar carência de investimentos em outros (Figura 2).

Nota-se na Figura 2 que os países que mais publicam estudos sobre o assunto são: Espanha, com 23,7\% das publicações, e Itália e Estados Unidos, com 21,1\% das publicações cada. Salienta-se, ainda, outros países importantes como: Inglaterra, China, Austrália, Canadá, Alemanha e Eslovênia; respectivamente em ordem crescente. Destaca-se ainda o Brasil, que consta na décima posição, empatando com Franca e Índia. 
Citação (APA): Abreu, V. H. S., de, Almeida, I. M., de, \& Turini, L. R. (2021). Utilização do transporte ativo para aumento da qualidade de vida da população urbana durante a pandemia do Covid-19 à luz de abordagem bibliométrica. Brazilian Journal of Production Engineering, 7(3), 139-152.

Figura 2. Publicações por País.

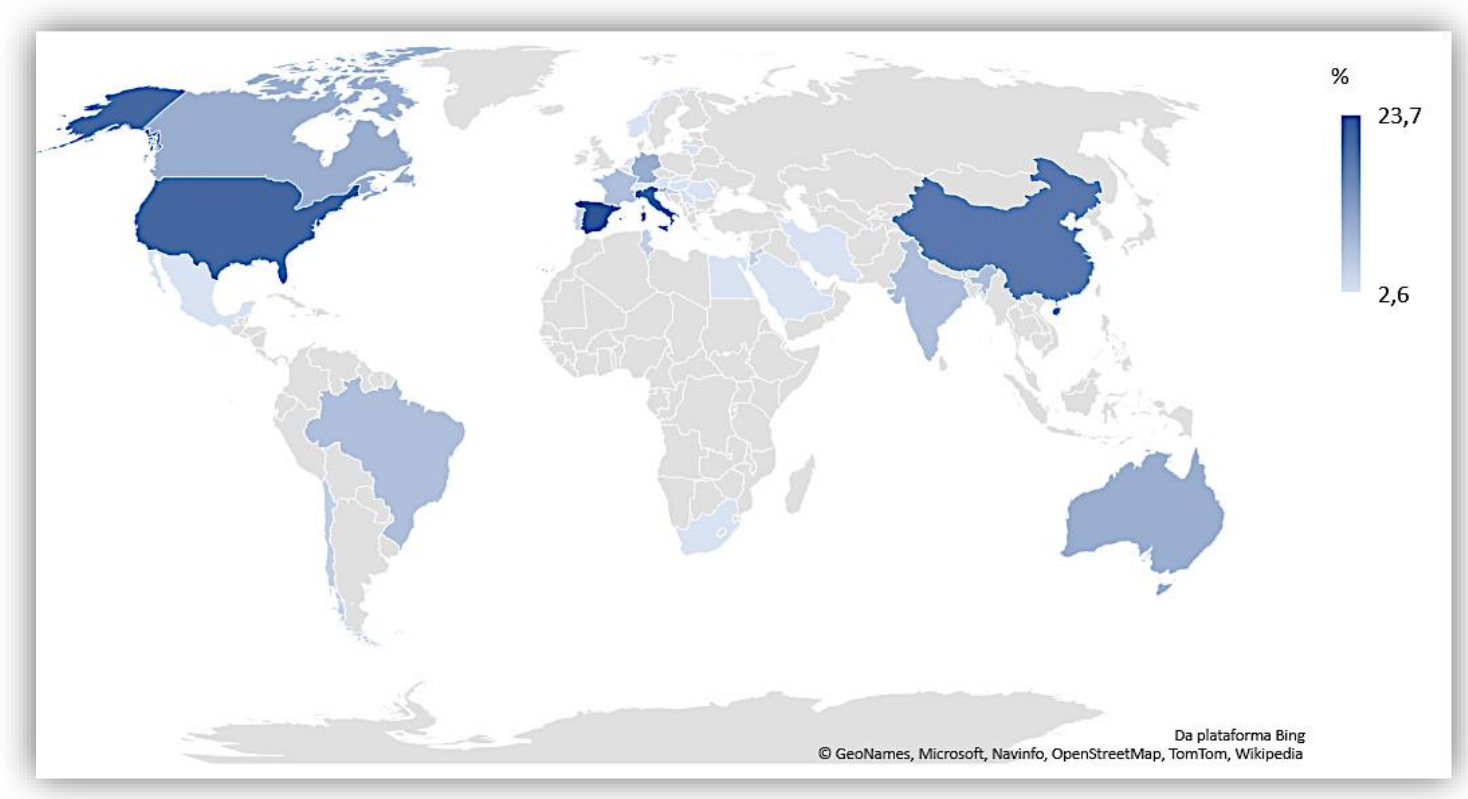

Fonte: Autores (2021).

Acredita-se também na importância de avaliar as principais palavras-chave encontradas nos artigos incluídos no repositório de pesquisa, conforme identificado na Figura 3, desenvolvida com auxílio do Software VOS Viewer. Essa estratégia permite que pesquisadores encontrem mais facilmente estudos diretamente relacionados ao assunto investigado, bem como identifiquem novos rumos de pesquisa.

Figura 3. Mapa de Calor da principais palavras-chave.

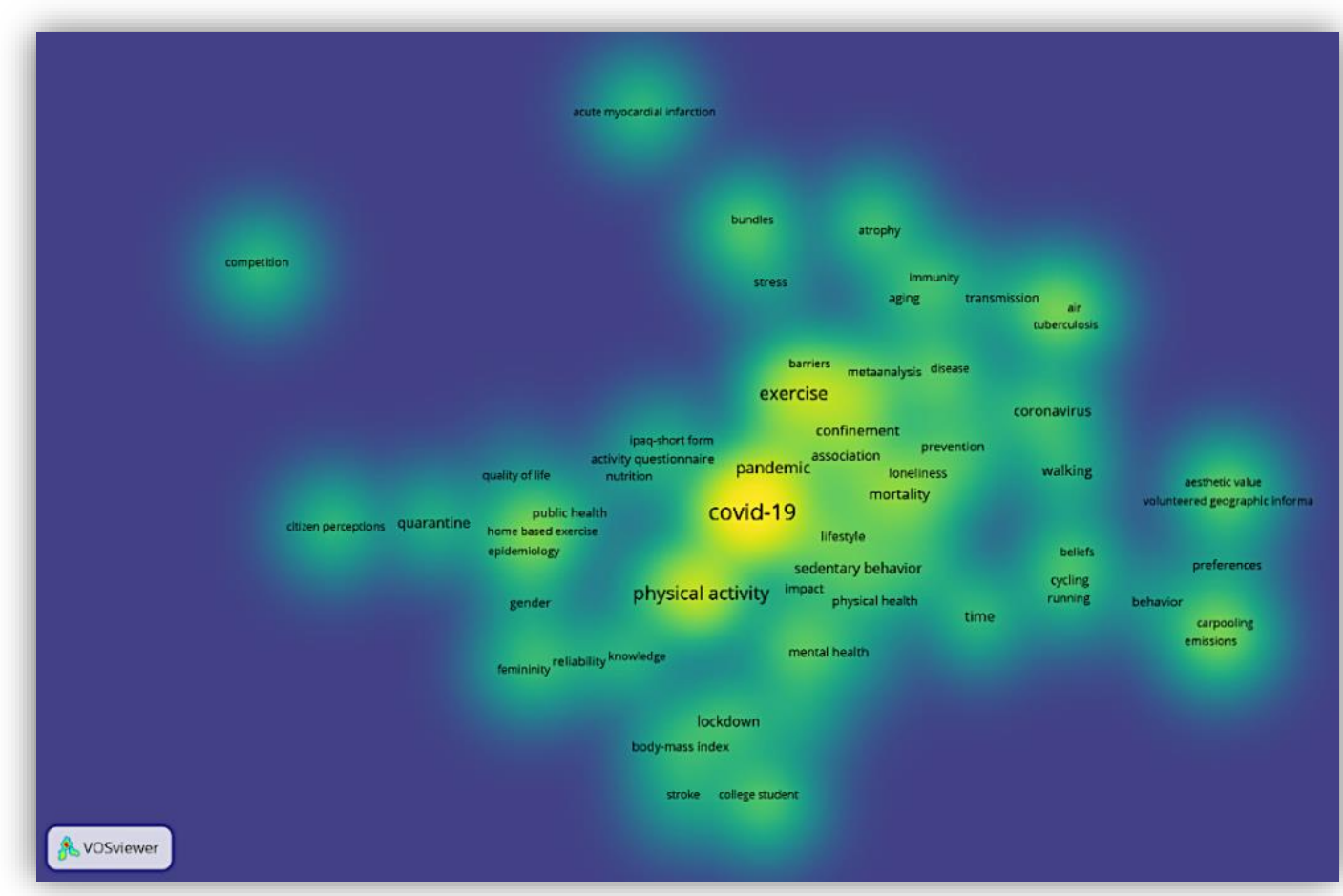

Fonte: Autores (2021).

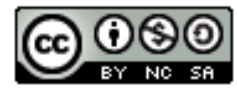

Esta obra está licenciada com uma Licença Creative Commons Atribuição-Não Comercial-Compartilha Igual 4.0 Internacional. Brazilian Journal of Production Engineering, São Mateus, Editora UFES/CEUNES/DETEC. 
Com o mapa de calor apresentado na Figura 3, nota-se que as palavras-chave com maior destaque foram: 'COVID-19'; 'Atividade Física' (do inglês, 'Physical-Activity'), 'Pandemia' (do inglês, 'Pandemic'); e 'Exercício' (do inglês, 'Exercise'). Além disso, pode ser dado destaque a outras palavras-chave com: 'Comportamento Sedentário' (do inglês, 'Sedentary Behavior'), 'Saúde Mental' (do inglês, 'Mental Health'), Mortalidade (do inglês, 'Mortality') e 'Qualidade de Vida' (do inglês, 'Quality of Life')

Ainda sobre as palavras-chave, é possível desenvolver uma rede de interligação entre elas, conforme apresentado na Figura 4, mais uma vez desenvolvida no Software VOS Viewer. A rede representada na Figura 4 é composta por 180 itens, 16 clusters e 1.214 links/conexões. Nela, é possível identificar as palavras-chave mais utilizadas (de acordo com o tamanho das esferas), bem como a ligação entre elas (de acordo com a cor das esferas e de suas interconexões).

Figura 4. Rede de interligação entre as principais palavras-chave.

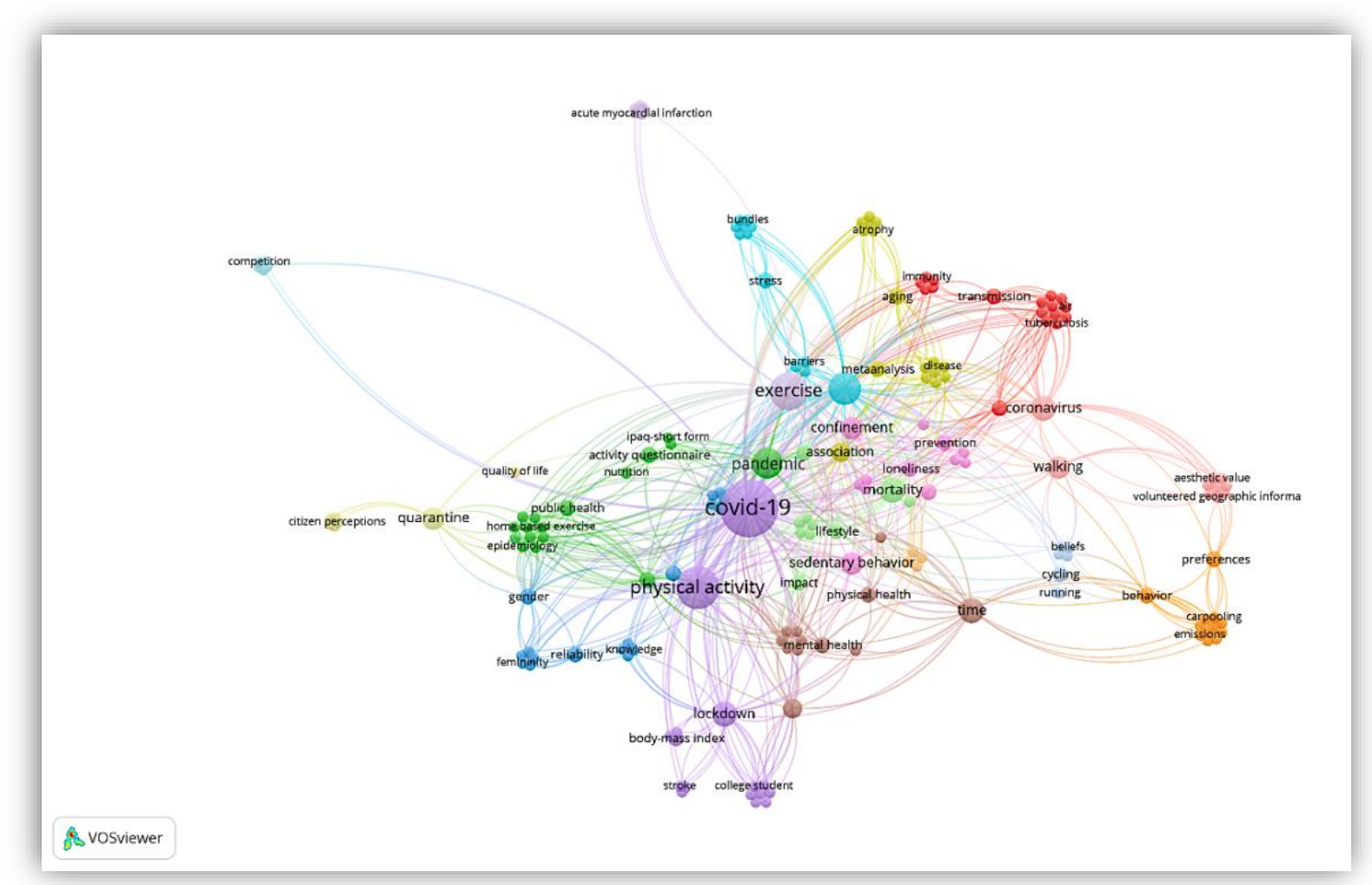

Fonte: Autores (2021).

Outra análise importante, refere-se ao número de citações por ano. Entretanto, assim como a evolução das publicações, as citações ocorrem apenas entre os anos de 2020 e 2021. Cabe ainda destacar, que mesmo sendo um assunto tão novo, os estudos incluídos no repositório de pesquisa já foram citados 222 vezes até o período de março de 2021.

Também é interessante identificar as áreas de aplicação dos estudos incluídos no repositório de pesquisa, conforme identificado na Tabela 3, em que $\mathrm{P}$ (Publicações) refere-se a porcentagem de artigos publicados sobre a área de interesse. Cabe mencionar, que um estudo

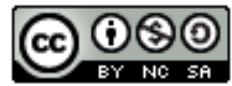


Citação (APA): Abreu, V. H. S., de, Almeida, I. M., de, \& Turini, L. R. (2021). Utilização do transporte ativo para aumento da qualidade de vida da população urbana durante a pandemia do Covid-19 à luz de abordagem bibliométrica. Brazilian Journal of Production Engineering, $7(3), 139-152$

pode se encaixar em mais de uma área de aplicação, por isso, o somatório de todas as áreas não corresponde a $100 \%$.

Tabela 3. Principais áreas de aplicação.

\begin{tabular}{cc}
\hline Áreas de pesquisa & P \\
\hline Ciências ambientais e ecologia & $24 \%$ \\
Saúde Pública, Saúde Ambiental e Ocupacional & $18 \%$ \\
Psicologia & $11 \%$ \\
Tecnologia científica e outros tópicos & $11 \%$ \\
Endocrinologia e metabolismo & $8 \%$ \\
Ciências e serviços de saúde & $8 \%$ \\
Nutrição e dietética & $8 \%$ \\
Pesquisa e Medicina Experimental & $8 \%$ \\
Ciência da Computação & $5 \%$ \\
Ciências do esporte & $5 \%$ \\
\hline
\end{tabular}

Fonte: Autores (2021).

Na Tabela 3, é possível identificar que as principais áreas são: Ciências ambientais e ecologia, com 24\% das publicações; Saúde Pública, Saúde Ambiental e Ocupacional, com 18\% das publicações; e Psicologia e Tecnologia científica e outros tópicos, com $8 \%$ das publicações cada. Salienta-se ainda, que todas as áreas de pesquisa identificadas apresentam uma relação direta ou indireta com a saúde física e mental.

Realizadas as análises bibliométricas, a Seção 4 tem como propósito destacar aspectos relacionados a utilização do transporte ativo para melhoria da saúde física e mental dos habitantes das cidades durante o período da pandemia do COVID-19.

\section{VISÃO GERAL SOBRE A IMPORTÂNCIA TRANSPORTE ATIVO DURANTE O COVID-19 COM FOCO EM QUALIDADE DE VIDA URBANA}

O COVID-19, que se iniciou em Wuhan, na China, teve rápida disseminação na cidade assumindo status de epidemia (Dutheil et al., 2020), onde no início de 2020 foram confirmados mais de 80 mil casos do vírus (Huang et al. 2020). Para evitar a disseminação para outros países, medidas como fechamento de fronteiras e restrições de deslocamento foram tomadas, mas essas estratégias não foram suficientes (Neto et al., 2020) e o vírus disseminou-se para outros países ao redor do globo, assumindo status de pandemia (Tosepu et al., 2020), acarretando uma enorme perturbação global com imensos impactos econômicos, ambientais e sociais em todo o mundo (Hendrickson \& Rilett, 2020; Wong et al., 2020).

Recomendações de saúde pública e medidas governamentais tomadas durante a pandemia do COVID-19 resultaram em inúmeras restrições na vida diária urbana, incluindo distanciamento social, isolamento e confinamento domiciliar (Arellana et al., 2020; Brady et al., 2020; de Vos, 2020; Ammar et al., 2020; WRI Brasil, 2020). Essas restrições tiveram um impacto generalizado na vida das pessoas, na maneira como se deslocam e no consumo de energia neste período (IEA, 2020). A supressão e mitigação dos impactos decorrentes da pandemia de COVID-19 são as principais prioridades para o futuro imediato; entretanto, também deve-se lidar com os custos sociais e de saúde decorrentes da redução da mobilidade populacional (BCHLA, 2020). 
Citação (APA): Abreu, V. H. S., de, Almeida, I. M., de, \& Turini, L. R. (2021). Utilização do transporte ativo para aumento da qualidade de vida da população urbana durante a pandemia do Covid-19 à luz de abordagem bibliométrica. Brazilian Journal of Production Engineering, $7(3), 139-152$.

Entre muitos outros setores, o transporte é um dos mais afetados, principalmente devido ao bloqueio no deslocamento das pessoas entre pontos de origem e destino (Muhammad et al., 2020; Moslem et al., 2020) e devido à possibilidade de rápida difusão geográfica do vírus através das rotas de transporte (Tian et al., 2020). Constata-se então que as viagens urbanas diminuíram em todo o mundo, especialmente as viagens pendulares e recreativas (Laverty et al., 2020; Abreu \& Turini, 2021). Com isso, os sistemas de transporte rodoviário foram reduzidos significativamente, tais como viagens em veículos privados e públicos (ônibus, metrôs, bondes, BRTs, etc.). Entre os impactos desta minimização na demanda por viagens motorizadas verificou-se, entre as externalidades positivas, a diminuição os níveis de congestionamento, o consumo de combustíveis fósseis e os acidentes de tráfego, por exemplo (Arellana et al., 2020).

As modificações de rotina impostas no dia a dia da população, resultante da pandemia COVID-19, podem ser vistas como uma oportunidade para implementar novos hábitos (Cheval et al., 2021), como um maior incentivo às viagens ativas (Nurse \& Dunning, 2020; Zhang et al., 2021; Souza \& Abreu, 2021), que se tornaram essenciais, não apenas para atender as necessidades básicas dos indivíduos, mas também para aumentar a qualidade de vida nas cidades (NCMM, 2020; Neto, 2020; Cheval et al., 2021). E, embora o isolamento seja uma medida necessária para proteger a saúde pública, pesquisas indicam que ele altera a atividade física e os comportamentos alimentares em um sentido comprometedor para saúde do indivíduo (Ammar et al., 2020). Com base nessas evidências científicas, manter uma rotina de exercícios regulares por meio de atividades de transporte ativo como caminhada, ciclismo e uso de patinetes é uma estratégia fundamental para aumentar a saúde física e mental dos habitantes das cidades durante um período de inatividade forçado, como a atual emergência do COVID-19 (Maugeri et al., 2020; Souza \& Abreu, 2021).

Isso porque a atividade física, promovida por meio das viagens ativas, reduz o risco de doenças cardiovasculares, vários tipos de câncer, demência, depressão e diabetes (Wannmacher, 2016; BCHLA, 2020; Brooks et al., 2020a), em especial durante períodos de confinamento, onde há uma maior probabilidade de desenvolver episódios de ansiedade e estresse, o que expõe ainda mais os indivíduos aos riscos à saúde (Brady et al., 2020). Complementarmente, verificou-se que, em pacientes com Artrite Reumatoide, as práticas de atividade física estão relacionadas à redução da ansiedade, depressão, fadiga, dor e aumento da vitalidade (Brooks et al., 2020b). Além disso, se prolongados, os efeitos negativos na saúde psicossocial e mental da população, os mesmos podem suprimir o sistema imunológico e as funções fisiológicas (Kiecolt-Glaser et al., 2002), o que pode aumentar o risco de exposição ao COVID-19 e a probabilidade de contrair a infecção (Slimani et al., 2020).

Dessa forma, o aumento da sustentabilidade e resiliência são esperados no futuro, mas devem ser apoiados por medidas eficazes de intervenção comportamental como o incentivo em larga escala e à longo prazo ao transporte ativo (Nurse \& Dunning, 2020; Souza \& Abreu, 2021). Felizmente, as viagens ativas podem crescer igualmente entre os gêneros, na maioria das faixas etárias e em todas as classes sociais, o que sugere o potencial para uma mudança social fundamental (Brooks et al., 2020a). 
Citação (APA): Abreu, V. H. S., de, Almeida, I. M., de, \& Turini, L. R. (2021). Utilização do transporte ativo para aumento da qualidade de vida da população urbana durante a pandemia do Covid-19 à luz de abordagem bibliométrica. Brazilian Journal of Production Engineering, $7(3), 139-152$.

Nesse sentido, governantes de diversas cidades pelo mundo estão incentivando a realização das viagens ativas em resposta ao vírus, por exemplo, por meio da construção de novas redes cicloviárias e fechamento de vias para realização de viagens ativas, de modo a incentivar opções fisicamente distantes de mobilidade pessoal que dificultem a disseminação do vírus (NCMM, 2020; Abreu \& Turini, 2021). Nesse aspecto, países como o Reino Unido e Dinamarca já criaram campanha de investimento para "nova era", onde foram inclusos no fundo ativo de emergencial, com a execução de vias de ciclismo e caminhada (Brooks et al., 2020a).

Embora a política e o financiamento para realização de alterações e expansões na infraestrutura viária que promovam o transporte ativo em nível macro sejam importantes, um aumento no deslocamento ativo provavelmente será mais difícil para alguns grupos, incluindo aqueles com longas distâncias de deslocamento, os socioeconomicamente desfavorecidos e os deficientes. Dessa forma, outras mudanças no ambiente físico em nível micro e ações sociais não devem ser negligenciadas (Brooks et al., 2020a), incluindo reestruturação ambiental, educação, capacitação, coerção e persuasão (Kelly et al., 2020). Cabe ainda destacar a necessidade de espaços urbanos verdes e acessíveis, principalmente para exercícios físicos, relaxamento e observação da natureza, trazendo assim efeitos benefícios para saúde física e mental dos habitantes (Ugolini et al., 2020).

\section{CONSIDERAÇÕES FINAIS}

Este artigo buscou realizar, por meio de revisão bibliográfica com abordagem bibliométrica, um apanhado geral dos estudos que investigam os aspectos que norteiam a utilização do transporte ativo com estratégia para melhoria da qualidade de vida da população urbana, durante o período da pandemia do COVID-19, o que também provocou fortes impactos na saúde física e mental de toda a sociedade.

Os resultados das análises bibliométricas mostram que o assunto, apesar de novo, tem despertado interesse crescente de pesquisadores e periódicos. A primeira publicação sobre o tema foi identificada no ano de 2020, o que era esperado devido ao fato da disseminação do COVID-19 ter sido mais abrangente no início deste ano em questão. E mesmo com a análise sobre as bases de dado desta pesquisa ter como limitação os meses anteriores a março de 2021, identificou-se diversos estudos sobre o tema sendo publicados nos primeiros três meses deste ano.

A pesquisa possibilitou a identificação da relevância do assunto a partir da observação que periódicos com elevado fator de impacto publicaram estudos sobre o assunto, como por exemplo, o Frontiers in Psychology, o International Journal of Obesity e o Sustainability. Além disso, verificou-se que os países que mais desenvolvem estudos sobre o assunto são a Espanha, a Itália e os Estados Unidos, que juntos concentram cerca de $66 \%$ de todas as publicações incluídas no repositório de pesquisa sobre o assunto.

A partir da elaboração de um mapa de calor foi possível observar as palavras-chave mais recorrentes. Além disso, também foi desenvolvida uma rede de interligação entre essas palavras-chave, o que possibilita que pesquisadores encontrem mais facilmente estudos

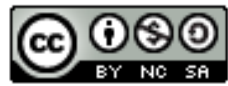


Citação (APA): Abreu, V. H. S., de, Almeida, I. M., de, \& Turini, L. R. (2021). Utilização do transporte ativo para aumento da qualidade de vida da população urbana durante a pandemia do Covid-19 à luz de abordagem bibliométrica. Brazilian Journal of Production Engineering, $7(3), 139-152$

diretamente relacionados ao assunto, bem como identifiquem novos rumos para a pesquisa. Nesta análise notou-se que as palavras com maior destaque relacionam o transporte ativo à pandemia de COVID-19 através da utilização destes modos na prática de atividades físicas. E é interessante observar que a pesquisa bibliométrica confirma esta influência do transporte ativo nos ganhos relacionados a saúde, tanto que as principais áreas de atuação identificadas estão relacionadas às ciências ambientais e a saúde em geral (física e mental).

Por fim, com a visão geral sobre a importância transporte ativo durante o COVID-19 apresentada, verificou-se que o setor de transporte foi um dos mais afetados durante a pandemia, especialmente devido à possibilidade de rápida difusão geográfica do vírus através das rotas de transporte, o que apontou o transporte ativo como uma solução para o deslocamento de curtas distâncias e, evidenciou, que as viagens ativas, através de práticas que incentivem as atividades físicas como caminhar e andar de bicicleta, são modos críticos de transporte que merecem mais atenção e financiamento. Nesse sentido, a pandemia do COVID-19 demostrou que, com a vontade dos tomadores de decisão de transportes e investimento financeiro dos setores público e privado, uma mudança significativa na matriz modal pode ocorrer mais rapidamente, trazendo segurança aos deslocamentos da população, promovendo bem-estar, qualidade de vida e saúde para a população.

Cabe destacar ainda que, como propostas de novos estudos sobre o assunto, aconselha-se que sejam desenvolvidas novas revisões bibliográficas com foco, por exemplo, na investigação sobre a equidade de acesso ao transporte ativo durante a pandemia do COVID-19, bem como sobre os efeitos a longo prazo das políticas de transporte implementadas durante a ocorrência da pandemia.

\section{Referências Bibliográficas}

Abreu, V. H. S., \& Turini, L. R. (2021). O transporte ativo no combate à disseminação do covid-19. Ciências da Saúde: uma abordagem pluralista. Editora Conhecimento Livre. Piracanjuba-GO. https://doi.org/10.37423/201203424

Alfonsin, N., McLeod, V., Loder, A., \& DiPietro, L. (2019). Evaluating a buildings' impact on active transportation: An interdisciplinary approach. Building and Environment, 106322. https://doi.org/10.1016/j.buildenv.2019.106322

Almeida, I. M., \& Abreu, V. H. S. (2021). Desafios para a Promoção do Transporte Ativo: uma Revisão da Literatura. In: XXI Congreso Panamericano de Transporte y Logística PANAM 2021, Lima, Peru.

Ammar, A., Brach, M., Trabelsi, K., Chtourou, H., Boukhris, O., Masmoudi, L., Bouaziz, B., Bentlage, E., How, D., Ahmed, M., Müller, P., Müller, N., Aloui, A., Hammouda O, Paineiras-Domingos LL, Braakman-Jansen A, Wrede C, Bastoni S, Pernambuco CS, Mataruna L, Taheri M, Irandoust K, Khacharem A, Bragazzi NL, Chamari K, Glenn JM, Bott, NT, Gargouri F, Chaari L, Batatia H, Ali GM, Abdelkarim O, Jarraya M, Abed KE, Souissi N, Van Gemert-Pijnen L, Riemann BL, Riemann L, Moalla W, Gómez-Raja J, Epstein M, Sanderman R, Schulz SV, Jerg A, Al-Horani R, Mansi T, Jmail M, Barbosa F, Ferreira-Santos F, Šimunič B, Pišot R, Gaggioli A, Bailey SJ, Steinacker JM, Driss T, Hoekelmann A. (2020). Effects of COVID-19 Home Confinement on Eating Behaviour and Physical Activity: Results of the ECLB-COVID19 International Online Survey. Nutrients, 28, 12(6), 1583. https://doi.org/10.3390/nu12061583

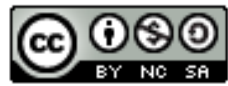


Citação (APA): Abreu, V. H. S., de, Almeida, I. M., de, \& Turini, L. R. (2021). Utilização do transporte ativo para aumento da qualidade de vida da população urbana durante a pandemia do Covid-19 à luz de abordagem bibliométrica. Brazilian Journal of Production Engineering, $7(3), 139-152$.

Arellana, J., Márquez, L., \& Cantillo, V. (2020). COVID-19 Outbreak in Colombia: An Analysis of Its Impacts on Transport Systems. Journal of Advanced Transportation, 1-16. https://doi.org/10.1155/2020/8867316

BBC - British Broadcasting Corporation. (2020). Will Covid-19 make urban cycling more inclusive? Disponível em https://www.bbc.com/worklife/article/20200724-will-covid-19make-urban-cycling-more-inclusive

BCHLA - BC Healthy Living Alliance. (2020). Guest post: BC Cycling Coalition's COVID19 and Active Transportation. Recuperado de https://www.bchealthyliving.ca/guest-post-bccycling-coalitions-covid-19-and-active-transportation/

Beck, M. J., \& Hensher, D. A. (2020). Insights into the impact of COVID-19 on household travel and activities in Australia - The early days under restrictions. Transport Policy, 96, 7693. https://doi.org/10.1016/j.tranpol.2020.07.001

Brady, S. M., Fenton, S. A. M., Metsios, G. S., Bosworth, A., Duda, J. L., Kitas, G. D., \& Veldhuijzen van Zanten, J. J. C. S. (2020). Different types of physical activity are positively associated with indicators of mental health and psychological wellbeing in rheumatoid arthritis during COVID-19. Rheumatology International. https://doi.org/10.1007/s00296-02004751-W

Brooks, J. H. M., Tingay, R., \& Varney, J. (2020a). Social distancing and COVID-19: an unprecedented active transport public health opportunity. British Journal of Sports Medicine, bjsports-2020-102856. https://doi.org/10.1136/bjsports-2020-102856

Brooks, S. K., Webster, R. K., Smith, L. E., Woodland, L., Wessely, S., Greenberg, N., \& Rubin, G. J. (2020b). The psychological impact of quarantine and how to reduce it: rapid review of the evidence. The Lancet. https://doi.org/10.1016/s0140-6736(20)30460-8

Chan, K., \& Farber, S. (2019). Factors underlying the connections between active transportation and public transit at commuter rail in the Greater Toronto and Hamilton Area. Transportation. https://doi.org/10.1007/s11116-019-10006-w

Chen, X. (2010). The declining value of subscription-based abstracting and indexing services in the new knowledge dissemination era. Serials Review, 36(2), 79-85.

Cheval, B., Sivaramakrishnan, H., Maltagliati, S., Fessler, L., Forestier, C., Sarrazin, P., Orsholits, D.; Chalabaev, A.; Sander, D; Ntoumanis, N. \& Boisgontier, M. P. (2021). Relationships between changes in self-reported physical activity, sedentary behaviour and health during the coronavirus (COVID-19) pandemic in France and Switzerland. Journal of sports sciences, 39(6), 699-704. https://doi.org/10.1080/02640414.2020.1841396

De Nazelle, A., Nieuwenhuijsen, M. J., Antó, J. M., Brauer, M., Briggs, D., BraunFahrlander, C., ... Lebret, E. (2011). Improving health through policies that promote active travel: A review of evidence to support integrated health impact assessment. Environment International, 37(4), 766-777. https://doi.org/10.1016/j.envint.2011.02.003

De Vos, J. (2020). The effect of COVID-19 and subsequent social distancing on travel behavior. Transportation Research Interdisciplinary Perspectives, 100121. https://doi.org/10.1016/j.trip.2020.100121

Dutheil, F., Baker, J. S., \& Navel, V. (2020). COVID-19 as a factor influencing air pollution? Environmental Pollution, 114466. https://doi.org/10.1016/j.envpol.2020.114466

Giménez-Gaydou, D. A., Cupido dos Santos, A., Mendes, G., Frade, I., \& Ribeiro, A. S. N. (2019). Energy consumption and pollutant exposure estimation for cyclist routes in urban areas. Transportation Research Part D: Transport and Environment, 72, 1-16. https://doi.org/10.1016/j.trd.2019.04.005

Hawkley, L. C., \& Capitanio, J. P. (2015). Perceived social isolation, evolutionary fitness and health outcomes: a lifespan approach. Philos. Trans. R. Soc. Lond. Ser. B Biol. Sci. 370:20140114. https://doi.org/10.1098/rstb.2014.0114 
Citação (APA): Abreu, V. H. S., de, Almeida, I. M., de, \& Turini, L. R. (2021). Utilização do transporte ativo para aumento da qualidade de vida da população urbana durante a pandemia do Covid-19 à luz de abordagem bibliométrica. Brazilian Journal of Production Engineering, $7(3), 139-152$

Hendrickson, C., \& Rilett, L. R. (2020). The COVID-19 Pandemic and Transportation Engineering. Journal of Transportation Engineering Part A: Systems, 146(7), 01820001. https://doi.org/10.1061/jtepbs.0000418

Huang, Y., Wu, Q., Wang, P., Xu, Y., Wang, L., Zhao, Y., Yao, D.; Xu, Y.; Ly, O. \& Xu, S. (2020). Measures undertaken in China to avoid COVID-19 infection: internet-based, crosssectional survey study. Journal of medical Internet research, 22(5) e18718. https://doi.org/10.2196/18718

IEA - International Energy Agency. (2020). Changes in transport behaviour during the Covid-19 crisis. Disponível em https://www.iea.org/articles/changes-in-transport-behaviourduring-the-covid-19-crisis

Kelly, P., Williamson, C., Baker, G., Davis, A., Broadfield, S., Coles, A., Gill, J. M. (2020). Beyond cycle lanes and large-scale infrastructure: a scoping review of initiatives that groups and organisations can implement to promote cycling for the Cycle Nation Project. British Journal of Sports Medicine, bjsports, 101447. https://doi.org/10.1136/bjsports-2019-101447

Kiecolt-Glaser, J. K., McGuire, L., Robles, T. F., \& Glaser, R. (2002). Emotions, morbidity, and mortality: new perspectives from psychoneuroimmunology. Annu. Rev. Psychol., 53, 83107. https://doi.org/10.1146/annurev.psych.53.100901.135217

Laverty, A. A., Millett, C., Majeed, A., \& Vamos, E. P. (2020). COVID-19 presents opportunities and threats to transport and health. Journal of the Royal Society of Medicine, 113(7), 251-254. https://doi.org/10.1177/0141076820938997

Maugeri, G., Castrogiovanni, P., Battaglia, G., Pippi, R., D’Agata, V., Palma, A., Musumeci, G. (2020). The impact of physical activity on psychological health during Covid-19 pandemic in Italy. Heliyon, 6(6), e04315. https://doi.org/10.1016/j.heliyon.2020.e04315

Moslem, S., Campisi, T., Szmelter-Jarosz, A., Duleba, S., Nahiduzzaman, K. M., \& Tesoriere, G. (2020). Best-Worst Method for Modelling Mobility Choice after COVID-19: Evidence from Italy. Sustainability, 12(17), 6824. https://doi.org/10.3390/su12176824

Muhammad, S., Long, X., \& Salman, M. (2020). COVID-19 pandemic and environmental pollution: A blessing in disguise? Science of The Total Environment, 138820. https://doi.org/10.1016/j.scitotenv.2020.138820

NCMM - National Center for Mobility Management. (2020). Active Transportation in the Era of COVID-19. Disponível em https://nationalcenterformobilitymanagement.org/activetransportation-during-covid-19/

Neto, T. O., Garcia, T. D. S. L., \& Spinussi, E. (2020). Pandemia de COVID-19, as fronteiras pelo mundo e o transporte aéreo na Itália. Confins. Revue franco-brésilienne de géographie/Revista franco-brasilera de geografia, 44. https://doi.org/10.4000/confins.27577

Nurse, A., \& Dunning, R. (2020). Is COVID-19 a turning point for active travel in cities? Cities \& Health, 1-3. https://doi.org/10.1080/23748834.2020.1788769

Rissel, C., Crane, M., Wen, L. M., Greaves, S., \& Standen, C. (2015). Satisfaction with transport and enjoyment of the commute by commuting mode in inner Sydney. Heal. Promot. J. Aust., 27, 80-83. https://doi.org/10.1071/HE15044

Rodrigues, P. F., Alvim-Ferraz, M. C. M., Martins, F. G., Saldiva, P., Sá, T. H., \& Sousa, S. I. V. (2019). Health economic assessment of a shift to active transport. Environmental Pollution, 113745. https://doi.org/10.1016/j.envpol.2019.113745

Rojas-Rueda, D., de Nazelle, A., Andersen, Z. J., Braun-Fahrländer, C., Bruha, J., BruhovaFoltynova, H., Nieuwenhuijsen, M. J. (2016). Health Impacts of Active Transportation in Europe. Plos One, 11(3), e0149990. https://doi.org/10.1371/journal.pone.0149990

Safe Routes Partnership. (2020). Advocating for Active Transportation in the Time of Covid19. Recuperado de https://www.saferoutespartnership.org/blog/advocating-active$\underline{\text { transportation-time-covid-19 }}$ 
Citação (APA): Abreu, V. H. S., de, Almeida, I. M., de, \& Turini, L. R. (2021). Utilização do transporte ativo para aumento da qualidade de vida da população urbana durante a pandemia do Covid-19 à luz de abordagem bibliométrica. Brazilian Journal of Production Engineering, $7(3), 139-152$

Sallis, J. F., Frank, L. D., Saelens, B. E., \& Kraft, M. K. (2004). Active transportation and physical activity: opportunities for collaboration on transportation and public health research. Transportation Research Part A: Policy and Practice, 38(4), 249-268. https://doi.org/10.1016/j.tra.2003.11.003

Santos, A. S., de Abreu, V. H. S., de Assis, T. F., Ribeiro, S. K., \& Ribeiro, G. M. (2021). An Overview on Costs of Shifting to Sustainable Road Transport: A Challenge for Cities Worldwide. Carbon Footprint Case Studies, 93-121. https://doi.org/10.1007/978-981-159577-6_4

Siedner, M. J., Harling, G., Reynolds, Z., Gilbert, R. F., Haneuse, S., Venkataramani, A. S., \& Tsai, A. C. (2020). Social distancing to slow the US COVID-19 epidemic: Longitudinal pretest-posttest comparison group study. PLOS Medicine, 17(8), e1003244. https://doi.org/10.1371/journal.pmed.1003244

Slimani, M., Paravlic, A., Mbarek, F., Bragazzi, N. L., \& Tod, D. (2020). The Relationship Between Physical Activity and Quality of Life During the Confinement Induced by COVID19 Outbreak: A Pilot Study in Tunisia. Frontiers in Psychology, 11. https://doi.org/10.3389/fpsyg.2020.01882

Souza, G. M.; Abreu, V. H. S. (2021). Transporte Ativo no Combate à Disseminação do COVID-19: Uma Abordagem Bibliométrica. In: XXI Congreso Panamericano de Transporte y Logística - PANAM 2021, Lima, Peru.

Tian H, et al. (2020). Early evaluation of Wuhan city travel restrictions in response to the 2019 novel coronavirus outbreak. Medrxiv. https://doi.org/10.1126/science.abb6105

Tosepu, R., Gunawan, J., Effendy, S.D., Ahmad, A.I., Lestari, H., Bahar, H., Asfian, P., (2020). Correlation between weather and Covid-19 pandemic in Jakarta, Indonesia. Sci. Total Environ. https://doi.org/10.1016/j.scitotenv.2020.138436

Ugolini, F., Massetti, L., Calaza-Martínez, P., Cariñanos, P., Dobbs, C., Ostoic, S. K., ... Sanesi, G. (2020). Effects of the COVID-19 pandemic on the use and perceptions of urban green space: an international exploratory study. Urban Forestry \& Urban Greening, 126888. https://doi.org/10.1016/j.ufug.2020.126888

Wannmacher, L. (2016). Obesidade como fator de risco para morbidade e mortalidade: evidências sobre o manejo com medidas não medicamentosas. Recuperado de https://www.paho.org/bra/index.php?option=com_docman\&view=download\&category_slug= serie-uso- racional-medicamentos-284\&alias=1535-obesidade-como-fator-risco-paramorbidade-e-mortalidade-evidencias-sobre-o-manejo-com-medidas-nao-

medicamentosas 5\&Itemid $=965$

Wong, A. Y.-Y., Ling, S. K.-K., Louie, L. H.-T., Law, G. Y.-K., So, R. C.-H., Lee, D. C.-W., Chung-Fai, Y.; Yung, P. S.-H. (2020). Impact of the COVID-19 pandemic on sports and exercise. Asia-Pacific Journal of Sports Medicine, Arthroscopy, Rehabilitation and Technology, 22, 39-44. https://doi.org/10.1016/j.asmart.2020.07.006

WRI Brasil. (2020). Bicicletas têm papel crucial na resiliência das cidades durante crise da Covid-19. Recuperado de https://wribrasil.org.br/pt/blog/2020/04/bicicletas-tem-papelcrucial-na-resiliencia-das-cidades- durante-crise-da-covid-19

Zhang, J., Hayashi, Y., \& Frank, L. D. (2021). COVID-19 and transport: Findings from a world-wide expert survey. Transport Policy, $103, \quad 68-85$. https://doi.org/10.1016/j.tranpol.2021.01.011 